Co-propagating Bose-Einstein condensates and electromagnetic radiation: formation of mutually localized structures

This article has been downloaded from IOPscience. Please scroll down to see the full text article.

2010 J. Phys. B: At. Mol. Opt. Phys. 43085301

(http://iopscience.iop.org/0953-4075/43/8/085301)

The Table of Contents and more related content is available

Download details:

IP Address: 163.1.243.11

The article was downloaded on 12/04/2010 at 13:08

Please note that terms and conditions apply. 


\title{
Co-propagating Bose-Einstein condensates and electromagnetic radiation: formation of mutually localized structures
}

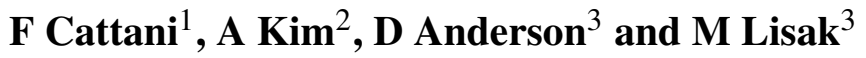 \\ ${ }^{1}$ Department of Physics, Clarendon Laboratory, Oxford OX1 3PU, UK \\ ${ }^{2}$ Institute of Applied Physics, Russian Academy of Sciences, 603950 Nizhny Novgorod, Russia \\ ${ }^{3}$ Department of Radio and Space Science, Chalmers University of Technology, SE-412 96 Göteborg, \\ Sweden \\ E-mail: f.cattani1@physics.ox.ac.uk
}

Received 23 October 2009, in final form 15 January 2010

Published 23 March 2010

Online at stacks.iop.org/JPhysB/43/085301

\begin{abstract}
A semi-classical model is derived for describing the interaction between coherent electromagnetic radiation and a Bose-Einstein condensate in the limit of zero temperature, including the back action of the atoms on the radiation. This model is used to analyse the problem of the self-consistent evolution of a laser beam and a BEC atomic beam. The mutual propagation is studied numerically and demonstrates not only the possibility of a stationary regime of mutual guiding, but also of generating a collapse-like phenomenon.
\end{abstract}

(Some figures in this article are in colour only in the electronic version)

\section{Introduction}

The interactions between electromagnetic radiation and neutral atoms, and in particular their nonlinear aspects, have found an intriguing protagonist with the realization of coherent atomic beams, strong Bosonic degeneracy being the reason why Bose-Einstein condensates (BEC) are playing a major role in this research field. When considering coherent atomic beams, the intrinsic nonlinearity related to atomatom interactions should be taken into account and, under the appropriate conditions, it can be described with a mathematical model strictly resembling that of the optical Kerr nonlinearity [1]. However, the presence of a light wave introduces more nonlinear features in the atom physics and we are interested in studying these effects from the point of view of atom manipulation and creation of localized light-atom structures.

The light wave acting as a nonlinear medium can mediate atomic interactions, in complete analogy with the role played by a nonlinear medium in the case of light nonlinearities. In the simplest model under dipole approximation, the electromagnetic radiation induces emission of dipole radiation which in turn gives rise to a long-range interaction among the atoms $[2,3]$. This model has attracted much interest since the pioneering work of Askar'jan [4] and Ashkin [5] who demonstrated how it was possible to describe and test the effects of electromagnetic radiation on neutral atoms, which made it possible to focus atoms with light. Klimontovich and Luzgin then proceeded in showing how neutral atoms can focus light as a result of light pressure dipole forces [6]. The main conceptual difference between those original models and the description of coherent atomic beams is due to the presence of long-range coherence as pointed out by Saffman [7] who modelled the contribution of light-mediated dipole-dipole interactions in terms of a contact potential while other authors worked out a description from first principles purely within the framework of quantum mechanics [8,9]. Efforts have also been made to go beyond the mean field approximation keeping into account as much as possible of the physics, including for example the effects of the inhomogeneity of the system and describing the physical effects that could be expected, see for instance [10]. Here we will consider a mean field model and in what follows, taking into account also the effects of the system inhomogeneity, we will give a description of the same phenomenon based on the analogy between matter waves and 
classical waves. We will then consider the possible formation of mutually localized structures and the possibility of having a collapse-like phenomenon even in one dimension.

\section{Semi-classical approach}

A semi-classical approach for describing the laser-induced atom interactions in a BEC was presented in [11], where the difference between the local field (the microscopic field acting on an atom) and the macroscopic field (the field averaged over a volume containing many atoms) was taken into account. Semi-classical reasoning leads to an extension of the Gross-Pitaevskii equation normally used to describe BECs through the definition of laser-induced forces (striction forces) which, written as gradient forces, yield a potential energy in agreement with fully quantum mechanical results. To calculate these forces, Helmholtz considered the work done by the electric field on a dielectric [12]. By working out the stress tensor through the variation of the dielectric free energy, an expression for the force was found for the case of a time-independent electric field [13]. This result was then generalized to the case of time-dependent fields by Pitaevskii [14] who showed that the expression for the stress tensor in this case is given by the time average of the same tensor calculated for a constant field. A straightforward application of Pitaevskii's results gives for the atom-atom interaction force induced by a far-off resonant laser field $\operatorname{Re}\left[\mathcal{E} \exp \left(-\mathrm{i} \omega_{L} t\right)\right]$ on a zero-temperature, dilute BEC:

$$
\mathbf{f}=\frac{n}{16 \pi} \nabla\left[|\mathcal{E}|^{2} \frac{\partial \epsilon}{\partial n}\right]
$$

where $n$ is the condensate atom density and $\epsilon$ is the dielectric permittivity of the condensate gas which has to be suitably modelled. Quantum theory and macroscopic electrodynamics [15] both yield

$$
\epsilon=1+\frac{4 \pi \alpha n}{1-\frac{4 \pi}{3} \alpha n},
$$

where, as derived from quantum theory, $\alpha=-d^{2} / \hbar \Delta$ is the atomic polarizability at the laser frequency, with $\Delta=$ $\omega_{L}-\omega_{a}$ being the detuning from the nearest atomic resonance frequency $\omega_{a}$, and $d$ is the dipole matrix element of the resonant transition. It is important to underline that (1) and (2) delimit the atomic physics we shall describe: the concept of force being a classical one, we will neglect quantum fluctuations, stochastic heating, incoherently scattered light and focus our attention on the coherent behaviour of the system. This limits the validity of the model mainly to large detunings $|\Delta| \gg$ $\omega_{a}, \Gamma$ (where $\Gamma$ is the atoms' natural line width). Besides, any singularity occurring within this model is unphysical since losses and saturation effects (which are not accounted for) would then become important. Neglecting these effects allows us to study possible mutual localization mechanisms and, in the eventuality of collapsing cases, this analysis will be relevant for the evolution stage occurring before saturation mechanisms become important. The character of long-range coherence is captured through the dipole-dipole interaction term.

The total force acting on a single atom is $\mathbf{F}=\mathbf{f} / n=$ $-\nabla V_{d}$ and the generalized Gross-Pitaevskii equation for the condensate wavefunction $\Psi(\mathbf{r}, t)$ in the spirit of mean field theory reads [1]

$$
\mathrm{i} \hbar \frac{\partial \Psi}{\partial t}=\hat{H}_{0} \Psi+\left[U_{0}|\Psi|^{2}-\frac{\alpha}{4} \frac{|\mathcal{E}|^{2}}{\left(1-\frac{4 \pi}{3} \alpha|\Psi|^{2}\right)^{2}}\right] \Psi .
$$

Here $\hat{H}_{0}$ is the linear single-particle Schrödinger Hamiltonian, the wavefunction $\Psi$ is normalized as $N=\int|\Psi|^{2} \mathrm{~d} \mathbf{r}$ with $N$ denoting the total number of atoms, so that the gas density is $n=|\Psi|^{2}, U_{0}=4 \pi \hbar^{2} a_{s} / m, m$ is the atom mass and $a_{s}$ is the $s$-wave scattering length which can be either positive or negative (repulsive or attractive collisional interaction). It must be kept in mind that this equation is valid for moderate laser intensities and for atom densities high enough to allow for a semi-classical description including near dipole-dipole interactions.

As already mentioned, effects of the laser-induced dipoledipole interactions have been considered for instance in [7] or [8], but with a simplified interaction term, justified by the relatively low atomic densities and large detunings considered, which allowed an expansion of the potential energy term for $|\Psi|^{2} \alpha \ll 1$. This is the most important difference between the present work and [7] since a low-density expansion makes the dipole interaction term independent of the sign of the detuning. It is natural, however, to expect that the structural dynamics of the condensate will depend on the nature of the nonlinearity, i.e. the sign of the detuning, and in the present investigation we will consider the fully nonlinear term. Depending on the sign of the detuning, the atom nonlinearity can have either a saturation character $(\Delta>0$, blue detuning, i.e. $\alpha<0)$ or a singularity character $(\Delta<0$, red detuning, i.e. $\alpha>0)$. By studying the consequences of the full nonlinear term on the density structures of an atomic beam copropagating with a laser beam, we expect to find an extension of wellknown results on cold atom guiding, see for instance [16] and references therein.

\section{Basic equations}

A first analysis which considered the full atomic nonlinearity but only a simplified description of the laser dynamics predicted the formation of self-localized atomic structures [11]. However, the feedback of the atoms on the laser was not taken into account and this effect may be of importance. In order to describe this feedback effect, it is necessary to include also a description of the evolution of the electromagnetic radiation, coupled to that of the atoms in order to analyse the possibility of mutual guiding and formation of localized structures.

The starting point is Maxwell's equations for propagation in a medium. Since we are interested in a stationary solution, we shall have

$$
\begin{gathered}
\mathbf{E}(\mathbf{r}, t)=\operatorname{Re}\left[\mathcal{E}(\mathbf{r}) \exp \left(-\mathrm{i} \omega_{L} t\right)\right] \\
\Psi(\mathbf{r}, t)=\Phi(\mathbf{r}) \exp \left(-\mathrm{i} \omega_{a} t\right)
\end{gathered}
$$

Under the assumption of $L_{n} \gg \lambda_{L}$ or $\nabla \epsilon \cdot \mathbf{E} \simeq 0$ where $L_{n}$ is the characteristic length scale of transverse density 
modulations and $\lambda_{L}$ is the radiation wavelength, we have from $\nabla \cdot \mathbf{D}=0$ that $\nabla \cdot \mathbf{E} \simeq 0$ and the wave equation

$$
\nabla \times \nabla \times \mathbf{E}+\frac{1}{c^{2}} \frac{\partial^{2} \mathbf{D}}{\partial t^{2}}=0,
$$

given (2), becomes $\left(\omega_{L}=k_{L} c\right)$

$$
\nabla^{2} \mathcal{E}+k_{L}^{2}\left(1+\frac{4 \pi \alpha|\Phi|^{2}}{1-\frac{4 \pi}{3} \alpha|\Phi|^{2}}\right) \mathcal{E}=0
$$

which corresponds to three scalar equations for the three components of the electromagnetic field.

This equation is further simplified by using the slowly varying envelope approximation, i.e. writing

$$
\begin{aligned}
& \mathcal{E}(\mathbf{r})=a\left(\mathbf{r}_{\perp}, z\right) \exp \left(\mathrm{i} k_{L} z\right) \mathbf{e}, \\
& \Phi(\mathbf{r})=\psi\left(\mathbf{r}_{\perp}, z\right) \exp \left(\mathrm{i} k_{a} z\right),
\end{aligned}
$$

where $\mathbf{r}_{\perp}$ denotes the dimensions transverse to the propagation direction $z$, $\mathbf{e}$ is the polarization vector of the field and $k_{a}$ is the atom wave number. The coupled system of equations (3), (7), can then be written in normalized variables as

$$
\begin{gathered}
\mathrm{i} \mu \frac{\partial \tilde{\psi}}{\partial \tilde{z}}=-\frac{1}{2} \tilde{\nabla}_{\perp}^{2} \tilde{\psi}+\frac{1}{2} \beta_{\text {coll }}|\tilde{\psi}|^{2} \tilde{\psi}-\frac{s}{2} \frac{|\tilde{a}|^{2}}{\left(1-s|\tilde{\psi}|^{2}\right)^{2}} \tilde{\psi} \\
\mathrm{i} \frac{\partial \tilde{a}}{\partial \tilde{z}}=-\frac{1}{2} \tilde{\nabla}_{\perp}^{2} \tilde{a}-\frac{3 s}{2} \frac{|\tilde{\psi}|^{2} \tilde{a}}{1-s|\tilde{\psi}|^{2}},
\end{gathered}
$$

where the following normalization has been used: $\tilde{\mathbf{r}}=\mathbf{r} k_{L}$, for the atom wavefunction $\tilde{\psi}=\psi / \psi_{*}$ with $(4 \pi|\alpha| / 3) \psi_{*}^{2}=1$, for the laser $\tilde{a}=a / a_{*}$, with $m|\alpha| a_{*}^{2} /\left(2 \hbar^{2} k_{L}^{2}\right)=1, s=\operatorname{sign}(\alpha)$, $\mu=k_{a} / k_{L}$ and $\beta_{\text {coll }}=6 a_{s} /\left(k_{L}^{2}|\alpha|\right)$. The tilde will be dropped hereafter unless otherwise stated.

If the assumption of a classical model for the laser field is justified by the choice of the intensity regime, it is clear that, for a mean field model to be valid based on the simplest possible approximation to the many-body atomic wavefunction, we must consider not only a zero-temperature limit but also a low density limit with $n a_{s}^{3} \ll 1$, see [17]. It will be shown hereafter that the parameter range in which we are interested is such as to satisfy this requirement.

We note that, although the laser equation is formally linear, it will nevertheless involve nonlinear evolution due to the presence of the $|\psi|^{2}$ term. For simplicity, in all studied cases we assume $\mu=1$ and consider one transverse dimension only, $r_{\perp}=x$, assuming that the system has a very large extension in $y$ and can be considered as homogeneous in that direction. It is possible to find exact localized, stationary solutions via the shooting method which will be discussed elsewhere [18]. Our main concern in the present investigation is how the initial transverse distributions of atom density $\left(|\psi(x, 0)|^{2}\right)$ and laser intensity $\left(|a(x, 0)|^{2}\right)$ vary during propagation along $z$.

\section{Laser and BEC atomic beam propagation}

From the point of view of physical effects, the most interesting case is that of an initially flat laser intensity profile where no gradient forces due to the electromagnetic radiation are present
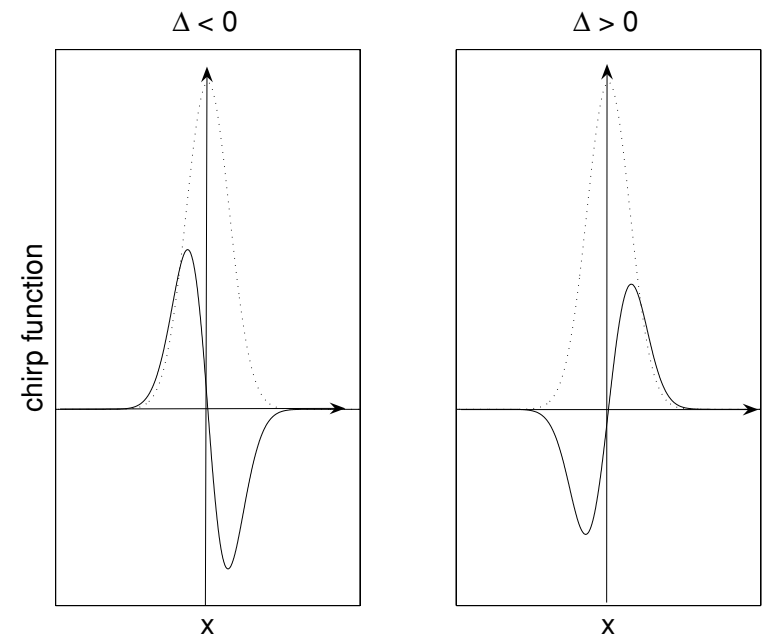

Figure 1. Qualitative behaviour of the chirp function (12), solid line, for an initial Gaussian atom density distribution, dashed line. Left: red detuning, i.e. $\operatorname{sign}(\alpha)>0$; right: blue detuning, i.e. $\operatorname{sign}(\alpha)<0$.

at the beginning of the propagation. As already pointed out by Saffman [7], spatial gradients can grow even on such an homogeneous intensity profile. However, in contrast with [7], we do not consider an explicit nonlinearity for the laser equation and in our case such gradients will be due to the effect of the atoms only. To gain insight on the physics acting during the very first stage of propagation, it is useful to look at the evolution of the laser intensity profile.

\subsection{Initial laser evolution}

In general, we may describe the propagating laser pulse amplitude as proportional to $\exp (\mathrm{i} h x+\mathrm{i} k z)$ where neither $h$ nor $k$ is known. The 'group velocity' of the pulse along $x$ during propagation is $v_{x}=-\partial k /\left.\partial h\right|_{h_{0}}$ (where $h_{0}$ is the central wave number in $x$, which is assumed to be zero). We expect the laser profile to diffract along $x$ during the pulse propagation along $z$ and the role of the atoms must be clarified. With an initially flat amplitude profile (e.g. super-Gaussian) the second derivative with respect to $x$ in (11) can be safely neglected. Thus, the presence of the atoms translates into a chirp on the laser transverse wave number as can be seen by separating real and imaginary parts in (11), discarding the second derivative and solving for the laser amplitude $|a|$ and phase $\varphi(a=|a| \exp (\mathrm{i} \varphi))$. The straightforward algebra gives $\varphi=2 \pi k_{L} \alpha|\psi|^{2} z /\left(1-4 \pi \alpha|\psi|^{2} / 3\right)$. The effect of such a chirp is well known in optics and it can be understood by noting that the modified phase $\varphi(x, z)$ induces a modification in the propagation velocity along $x$ of the different Fourier components of the pulse. In fact, we can now write for the propagating pulse $|a| \exp (\mathrm{i} \varphi(x)+\mathrm{i} k z)$ which shows how the chirp effect is to create an instantaneous $x$-wave number [20]

$$
h_{c}=\frac{\partial \varphi}{\partial x}=\frac{2 \pi \alpha k_{L} z}{\left(1-\frac{4 \pi}{3} \alpha|\psi|^{2}\right)^{2}} \frac{\partial|\psi|^{2}}{\partial x},
$$

i.e. $h=h_{0}+h_{c}$. Assuming an initial Gaussian form for the atom wavefunction well localized within the laser intensity distribution, the qualitative behaviour of the chirp 

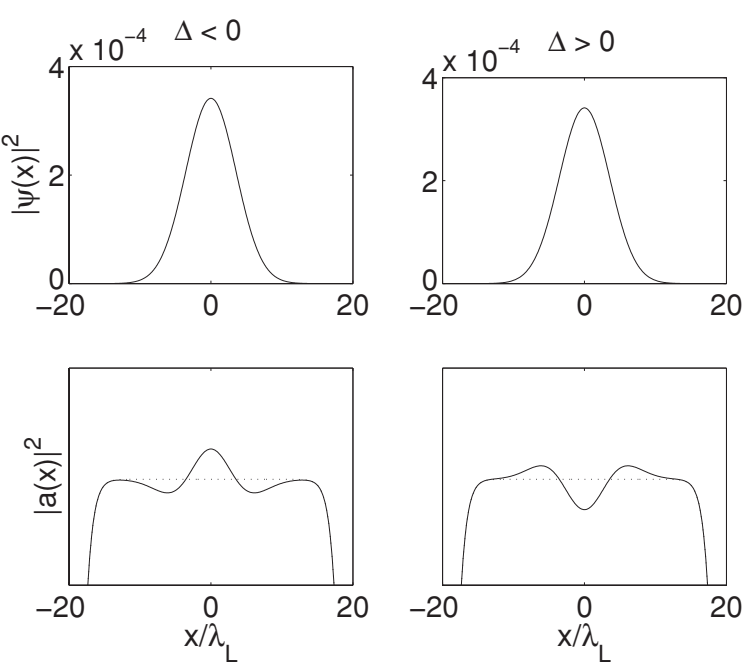

Figure 2. Atom density and laser intensity (details of flat top) for red detuning (left) and blue detuning (right) after a very short propagation $\left(z=0.0012 \lambda_{L}\right)$. Dotted line: initial intensity distribution. The parameters are the same as for run $a$, see the text. Normalization as given in the text.

as a function of $x$ is shown in figure 1. Since we have ${ }^{4}$ $\mathrm{d} v_{x} / \mathrm{d} h=-\partial^{2} k /\left.\partial h^{2}\right|_{h_{0}}>0$, parts of the pulse with $h>h_{0}$ will have a higher propagation velocity along $x$ and vice versa. Looking at figure 1, we expect that those parts of the pulse that are localized around the positive peak of the chirp function will move with higher velocities in the direction of positive $x$ while those parts of the pulse that are localized around the negative peak will slow down. In the red detuning case, this creates a central peak in the laser intensity profile and two symmetric troughs where the chirp effect is stronger and empties the pulse. This is clearly seen in the numerical simulations immediately after the simulations start. In the blue detuning case, the result of the chirp effect is the opposite; thus, the seed for further evolution depends on the sign of the detuning. An example of the modification induced on the laser intensity profile can be seen in figure 2 for two runs with the same initial conditions but opposite sign of the detuning. Once this initial change in the laser structure is formed, it drives a dynamical reaction on the atom density since the potential now felt by the atoms has been modified. Again, the response will be different according to the sign of the detuning.

\subsection{Red detuning}

This is an interesting case since the atoms will feel a focusing action from the dipole-induced potential. In particular, such an action will be strengthen by the initial formation of a central peak in the laser intensity and may lead to a catastrophic instability with subsequent collapse even in the one-dimensional case. As noted above, the atom equation has a singular nonlinearity and we may define a critical atom density as $\psi_{*}^{2}=3 /(4 \pi|\alpha|)$ in unnormalized variables (i.e,

4 As can be seen by Taylor-expanding $k=k\left(h_{0}\right)+\partial k /\left.\partial h\right|_{h_{0}}\left(h-h_{0}\right)+$ $1 / 2 \partial^{2} k /\left.\partial h^{2}\right|_{h_{0}}\left(h-h_{0}\right)^{2}+\cdots$ According to the usual transformation $\mathrm{i} k \leftrightarrow \mathrm{i} \partial / \partial z$ from the expansion for $k$ we obtain an equation for the laser amplitude which corresponds exactly to our (11) if $\partial^{2} k /\left.\partial h^{2}\right|_{h_{0}}<0$. $\psi_{*}^{2}=1$ normalized variables), dependent on the detuning and on the dipole moment. However, the presence of the collisional nonlinearity cannot be neglected and should play an even more important role as soon as the atom density undergoes focusing. For a negative scattering length, the more the atoms are focused, the stronger the focusing action and this is expected to strictly limit the stability of the system against collapse, whereas a repulsive collisional nonlinearity, acting in the same direction as the diffractive kinetic energy contribution, should tend to balance the focusing effect of the laser-induced nonlinearity. The possibility of reaching this equilibrium (i.e. a structure consisting of two stationary localized coupled solutions) depends on the initial conditions and, to our knowledge, cannot be studied analytically. We have investigated numerically the parameter range for stability/collapse at fixed initial width of both the superGaussian laser intensity and the Gaussian atom density profile given as

$$
\begin{aligned}
& \psi(x, 0)=\psi_{0} \mathrm{e}^{\left(-x^{2} / 2 d_{a}^{2}\right)} \\
& a(x, 0)=a_{0} \mathrm{e}^{-\left(x^{2} / 2 d_{L}^{2}\right)^{g}}
\end{aligned}
$$

where $g$ is the super-Gaussian parameter $(g=10$ in the simulations).

In order to describe the numerical results it is useful to present a reference case with parameters such that the system settles down rather smoothly onto a stationary, mutually localized structure for both atom density and laser intensity. A possible such case (the one referred to as run $(a)$ ) has $d_{a}=$ $5 \lambda_{L}, d_{L}=40 \lambda_{L}$, initial peak atom density $5.81 \times 10^{-18} \mathrm{~m}^{-3}$ (corresponding to $\tilde{\psi}_{0}^{2}=3.41 \times 10^{-4}$ ), initial peak laser intensity $0.0153 \mathrm{~mW} \mathrm{~cm}^{-2}$ (corresponding to $\tilde{a}_{0}^{2}=0.0181$ ), with $\beta_{\text {coll }}=38$ which corresponds for instance to a detuning of 100 times the decay rate for ${ }^{87} \mathrm{Rb}$ atoms. From these initial conditions, the system stabilizes onto the localized structures shown in figure 3 , with the evolution of the peak atom density and laser intensity showing characteristic relaxation oscillations during the approach to the final stationary state, see figure 4. The atom transient dynamics is governed by an interplay between their kinetic energy and interatomic repulsive interactions together with the focusing laser-induced potential. The atoms initially see a potential trap induced by the presence of the laser combined to a central repulsive barrier due to their own repulsive interaction, see the inset in figure 3. The natural thing to happen is for the atoms to escape from the central region where they are initially localized. The increase in the peak laser intensity (chirp effect) and the decrease in repulsion (decrease in the central atom density) both tend to create a central trap where initially there was a repulsive barrier, with the effect that some atoms remain centrally localized while those spreading out will move to encounter the lateral walls of the laser-induced potential trap.

The laser intensity profile tends to evolve according to what known for super-Gaussian pulses (note that initially $d_{L} \gg d_{a}$ ) [21], forming modulations on its shoulders which in turn modify the laser-induced potential trap thus affecting the natural broadening of the atom wavefunction. Some atoms, 

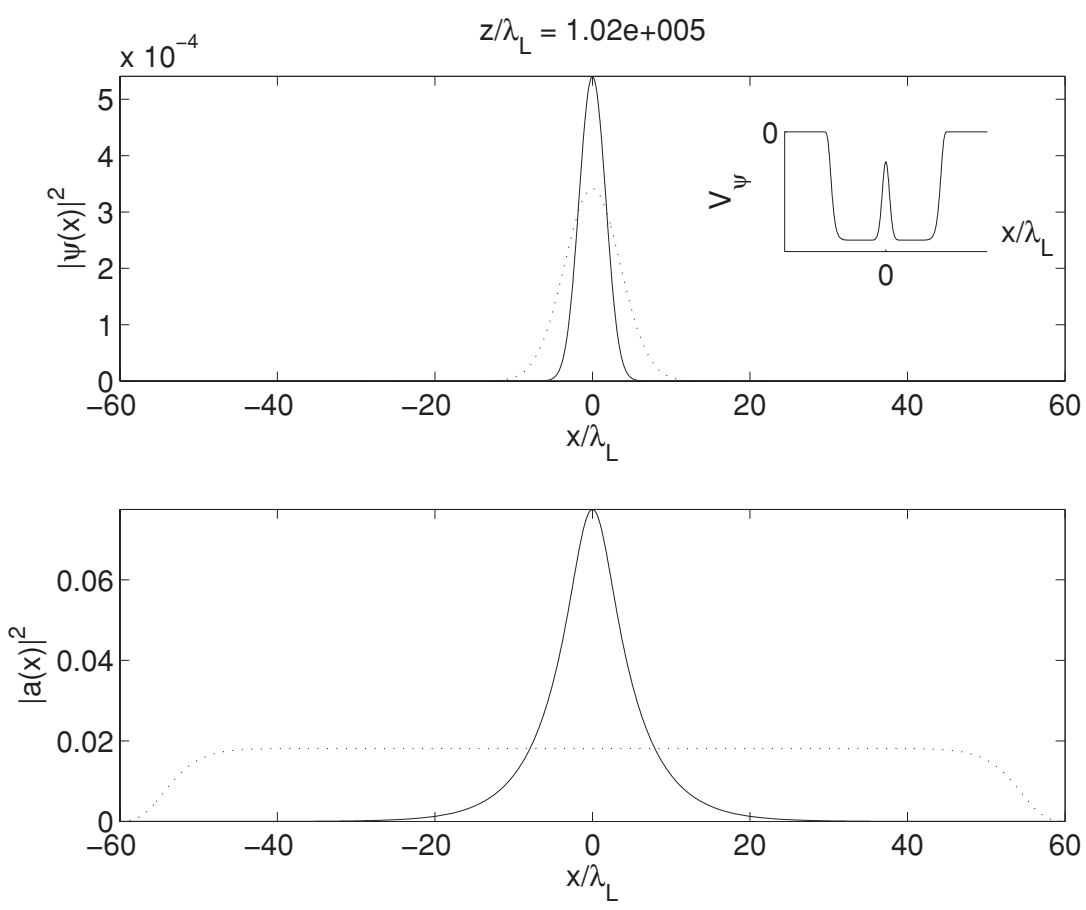

Figure 3. Stationary atom density and laser intensity after $1.02 \times 10^{5} \lambda_{L}$ of propagation along $z$ for run $(a)$. Dotted lines: initial distributions. Normalization as in the text. The inset shows the potential initially felt by the atoms.
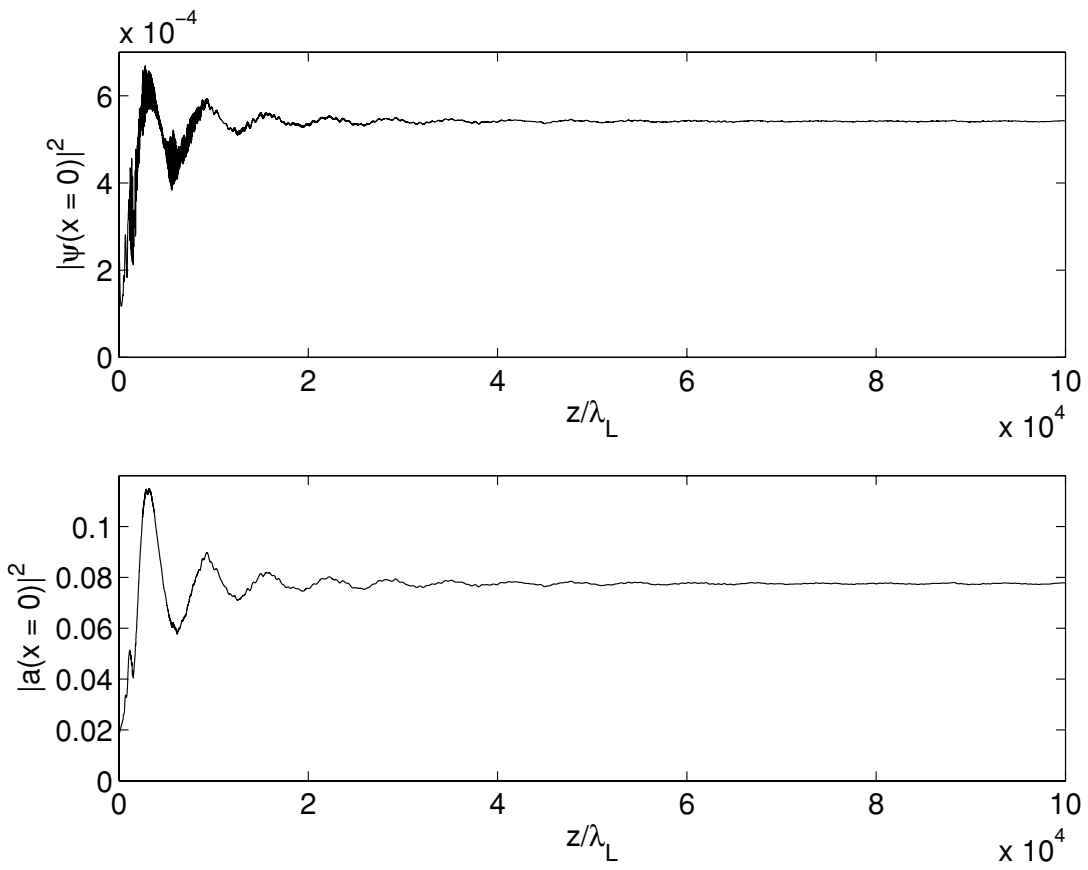

Figure 4. Peak atom density and laser intensity during propagation along $z$ for run $(a)$. Normalization as in the text.

and part of the electromagnetic radiation too, will escape out of the laser-induced trap but most of them in this case remain trapped and return back to interact with the central bunch. This process continues until a final stationary state is reached. Figure 5 shows some snapshots of this evolution. The resulting structures have proved to be extremely robust against numerical perturbation tests.

Although, for fixed $d_{L} / d_{a}$, the physics of the laser evolution (i.e. central chirp, lateral modulation and shoulder formation) is qualitatively the same, the nature of the transient for the atoms changes depending on $\psi_{0} / a_{0}$ since the balance between the different effects changes as can be seen by looking at the initial potential felt by the atom wavefunction, see figure 6.

Increasing the initial atom peak density, with fixed initial laser peak intensity, leads to the formation of mutually localized structures with higher and higher peak density, see figure 7(A). These results also give a good idea of what sort of physical parameters can be expected for these structures. For the initial conditions given in the caption of figure $7(\mathrm{~A})$ 

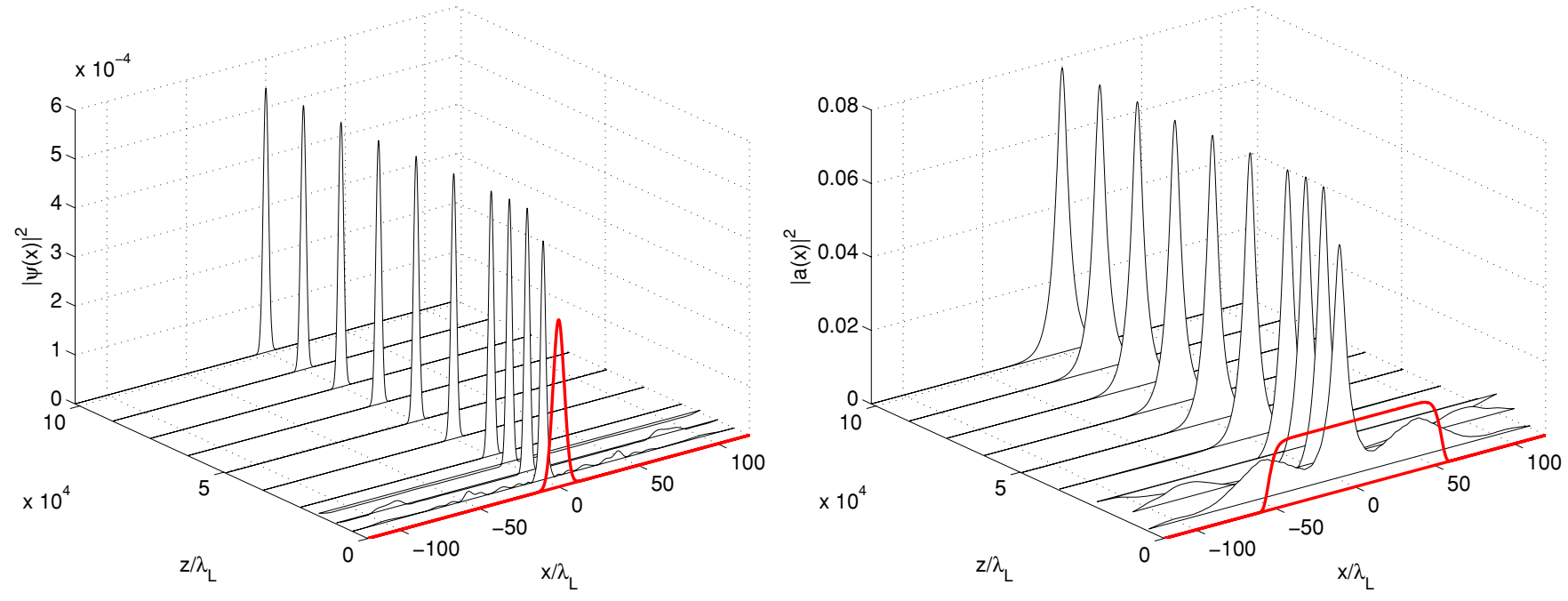

Figure 5. Snapshots of the evolution of atom and laser wavefunctions during propagation along $z$ for run $(a)$. The first curve (red online) is the initial wavefunction. Normalization as in the text.

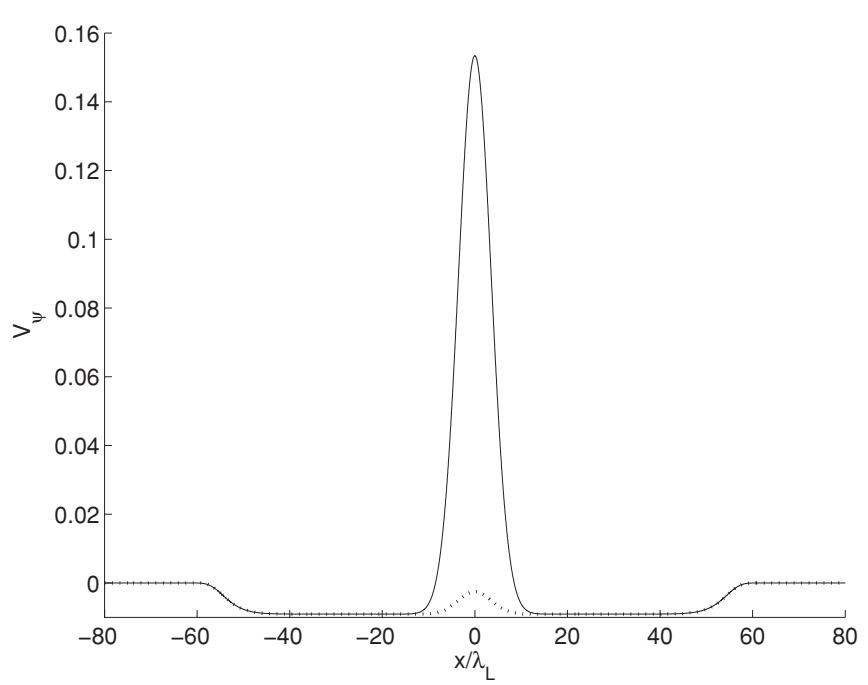

Figure 6. Initial potential experienced by the atom wavefunction for two different initial peak densities: $n_{0}=5.81 \times 10^{18} \mathrm{~m}^{-3}$ (same as run $(a), \tilde{\psi}_{0}=0.0185$, dotted line), $n_{0}=1.44 \times 10^{20} \mathrm{~m}^{-3}$ $\left(\tilde{\psi}_{0}=0.092\right.$, solid line). The initial peak laser intensity and all other parameters are the same as for run $(a)$. Normalization as in the text.

and an initial red detuning of about $3.81 \mathrm{GHz}$, i.e. 100 times the decay rate of ${ }^{87} \mathrm{Rb}$ of the $5^{2} \mathrm{~S}_{1 / 2}-5^{2} \mathrm{P}_{3 / 2}$ atomic resonance, the peak atom densities and peak laser intensities tend to settle down to values of the order of $10^{-19} \mathrm{~m}^{-3}$ and $0.06 \mathrm{~mW} \mathrm{~cm}^{-2}$. The maximum peak atom densities and peak laser intensities reached during this evolution process are of the order of $1.8 \times 10^{19} \mathrm{~m}^{-3}$ and $0.1 \mathrm{~mW} \mathrm{~cm}{ }^{-2}$ respectively. Thus, even at the maximum values reached, the low-density condition $n a^{3} \ll 1$ still holds, being $n a^{3} \sim 10^{-5}-10^{-6}$ for all cases of figure 7 .

In the model we have presented, all absorption processes were neglected, a legitimate assumption provided that the laser detuning $\Delta$ is so large compared with the spontaneous emission rate that the imaginary part of the dielectric permittivity can be considered negligibly small. In this case the effect of resonance absorption on the BEC density modulations is small but will define the life-time of these structures. Even at very large detunings, resonance absorption could come into play due to photoassociation which can be an effective mechanism of excitation of the high-lying vibrational levels of an excited molecule created from two atoms during a collisional process [22]. However, photoassociation spectra are in general known to be quite narrow, and comparing the range of detunings we are considering here with experimental data for photoassociation spectra, see for instance [23], shows that it is possible to neglect photoassociation effects in the parameter range considered for our model.

For too high initial values of $\tilde{\psi}_{0}$ we have found a new regime and a qualitatively different stationary state with the creation of two symmetric, mutually localized structures, moving outwards, see figure 8 . This is the result of having initially a central repulsive push not counterbalanced by the action of the laser in spite of the enhancement due to the chirp effect. The formation, dynamics and characteristics of these jet-like structures will be discussed elsewhere. A further increase in $\psi_{0}$ yields a dominant broadening of the atom laser function and a monotonic decrease of the peak atom density.

Increasing the initial laser peak intensity for a fixed initial atom density also makes it possible to generate a continuous family of mutually localized solutions, see figure 7(B) with examples of final stationary structures shown in figure 9.

However, this also opens up the possibility of a collapselike instability when the initial laser intensity is larger than a critical value which depends on the initial atom density. We have identified as collapsing cases, within the approximations of this model, those cases in which the peak atom density showed an increase towards the critical value corresponding to $\tilde{\psi}_{0}^{2}=1$, i.e. $n_{*}=1.7 \times 10^{22} \mathrm{~m}^{-3}$. From the numerical point of view, the propagation step required to maintain the error within given limits decreases to zero. There seems to be a threshold intensity such that, above this, the diffractive contributions to the atom dynamics can no longer balance the focusing effect of 

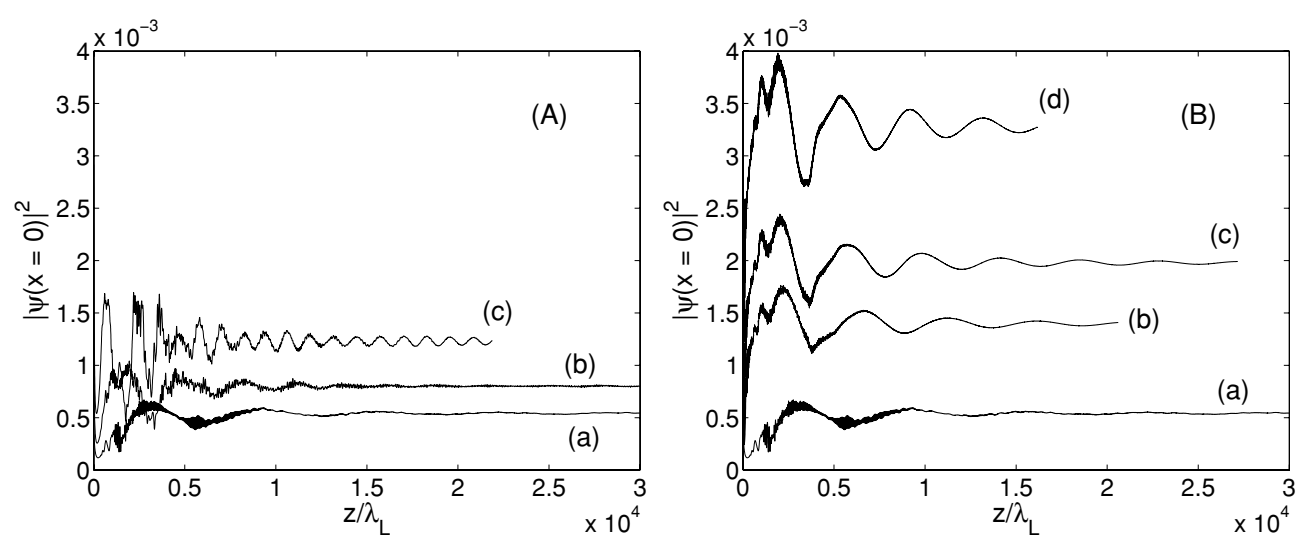

Figure 7. (A) Atom peak densities during propagation for fixed laser peak intensity $\tilde{a}_{0}=0.1346, I_{0}=0.0153 \mathrm{~mW} \mathrm{~cm}^{-2}$ and

(a) $\tilde{\psi}_{0}=0.0185, n_{0}=5.81 \times 10^{18} \mathrm{~m}^{-3}$, (b) $\tilde{\psi}_{0}=0.0316, n_{0}=1.7 \times 10^{19} \mathrm{~m}^{-3}$, (c) $\tilde{\psi}_{0}=0.054, n_{0}=4.96 \times 10^{19} \mathrm{~m}^{-3}$. (B) Atom peak densities during propagation for fixed atom peak density $\tilde{\psi}_{0}=0.0185, n_{0}=5.81 \times 10^{18} \mathrm{~m}^{-3}$ and (a) $\tilde{a}_{0}=0.1346, I_{0}=0.0153 \mathrm{~mW} \mathrm{~cm}$, (b) $\tilde{a}_{0}=0.3873, I_{0}=0.127 \mathrm{~mW} \mathrm{~cm}^{-2}$, (c) $\tilde{a}_{0}=0.5477, I_{0}=0.254 \mathrm{~mW} \mathrm{~cm}^{-2}$, (d) $\tilde{a}_{0}=1, I_{0}=0.847 \mathrm{~mW} \mathrm{~cm}^{-2}$. Normalization as in the text.
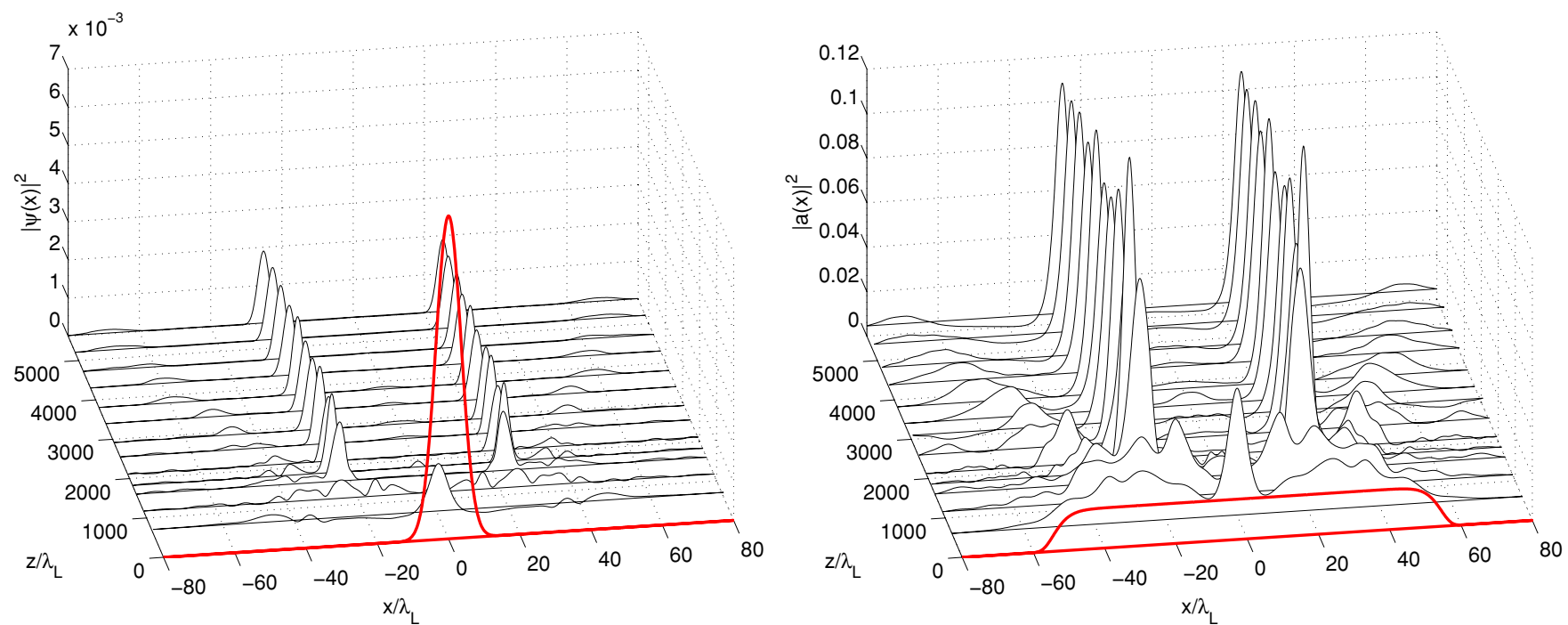

Figure 8. Structures formed starting from the initial peak density $n_{0}=1.44 \times 10^{20} \mathrm{~m}^{-3}$ (corresponding to the solid line potential shown in figure 6). All other parameters are the same as for run $(a)$. Normalization as in the text.
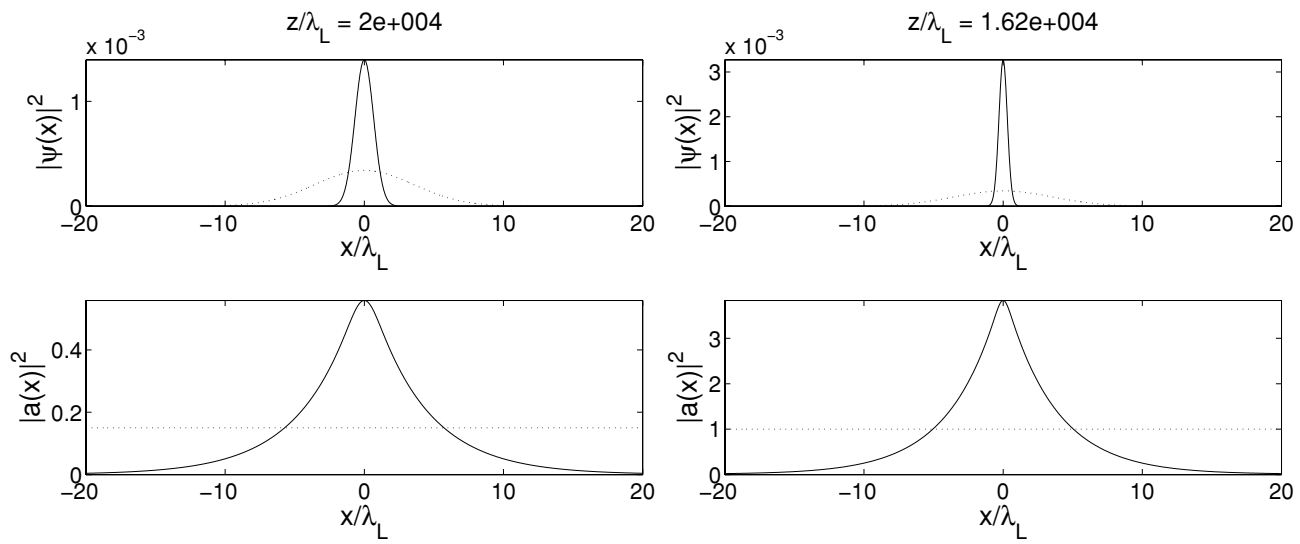

Figure 9. Left column: stationary atom density and laser intensity after $1.99 \times 10^{4} \lambda_{L}$ of propagation along $z$ for run (b) of figure 7(B). Right column: after $1.61 \times 10^{4} \lambda_{L}$ of propagation along $z$ for run (d) of figure 7(B). Dotted lines: initial distributions. Normalization as in the text. 

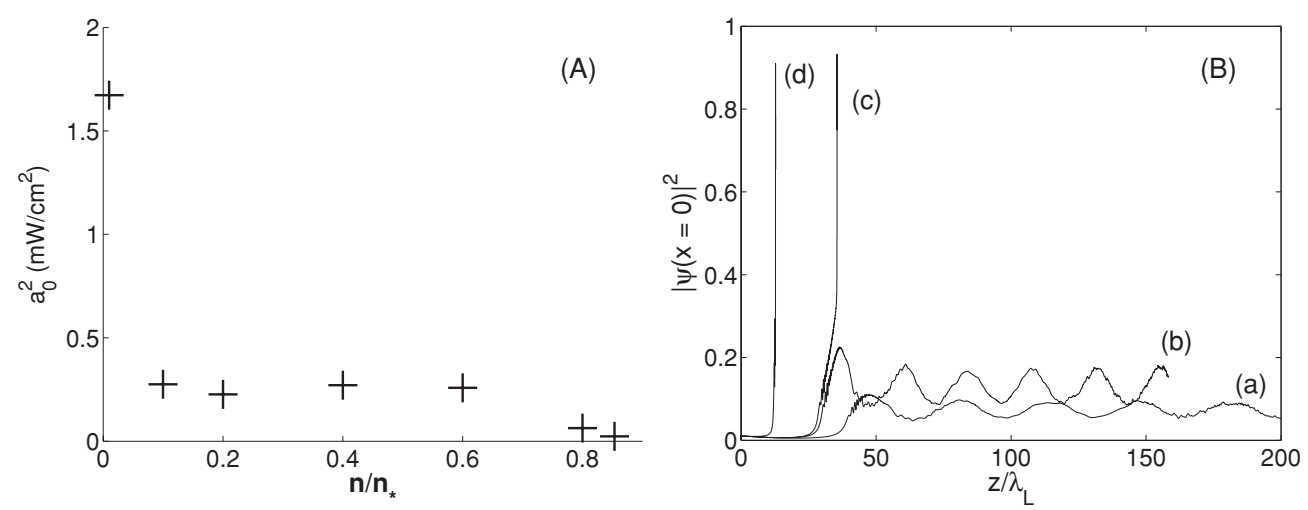

Figure 10. (A) Minimum initial laser intensity at which collapse was numerically observed for different values of the initial atom density $n_{0}$ given with respect to the critical one $n_{*}$. (B) Peak atom densities during the propagation for varying initial laser intensities. For all cases $\tilde{\psi}_{0}=0.1, n_{0}=1.7 \times 10^{20} \mathrm{~m}^{-3}$ and (a) $\tilde{a}_{0}=1, I_{0}=0.847 \mathrm{~mW} \mathrm{~cm}^{-2}$, (b) $\tilde{a}_{0}=1.8, I_{0}=1.52 \mathrm{~mW} \mathrm{~cm}^{-2}$, (c) $\tilde{a}_{0}=1.975, I_{0}=$ $1.67 \mathrm{~mW} \mathrm{~cm}^{-2}$, (d) $\tilde{a}_{0}=10, I_{0}=8.47 \mathrm{~mW} \mathrm{~cm}^{-2}$. Normalization as in the text.

the dipole-dipole interaction. Furthermore, due to the singular character of the focusing nonlinearity, this threshold decreases for higher initial atom densities. The numerically observed threshold intensities for collapse are given in figure 10(A) for a range of initial atom densities. It is plausible that for low atom densities no collapse occurs, irrespective of how large the laser intensity is; however, the corresponding extremely rapid dynamical features observed cannot be described under the hypothesis of the slowly varying envelope approximation. The effect of lowering the laser intensity is shown in figure 10 (B) for a particular value of the initial atom density.

\section{Conclusion}

The present analysis has demonstrated that it should be possible to exploit the interaction of coherent electromagnetic radiation and BEC atomic beams to generate mutually localized structures. Assuming an initial flat-top profile for the laser intensity, the final peak densities and intensities, as well as the widths of the generated structures, depend on the initial conditions. It also seems possible to induce a collapselike phenomenon on these systems. However, in such cases the model used here is insufficient and must be generalized to incorporate additional effects that are neglected in the present analysis.

\section{Acknowledgment}

FC would like to acknowledge the hospitality offered by the Department of Radio and Space Physics of Chalmers University of Technology during the preparation of this work.

\section{References}

[1] Dalfovo F, Giorgini S, Pitaevskii L P and Stringari S 1999 Rev. Mod. Phys. 71 463-512

[2] Cohen-Tannoudji C, Dupont-Roc J and Grynberg G 1998 Atom-Photon Interactions: Basic Processes and Applications (Berlin: Wiley)
[3] Craig D P and Thirunamachandran T 1984 Molecular Quantum Electrodynamics (London: Academic)

[4] Askhar'yan G A 1962 Zh. Eksp. Teor. Fiz. 421567 Askhar'yan G A 1962 Sov. Phys_JETP 151088 (Engl. Transl.)

[5] Ashkim A 1970 Phys. Rev. Lett. 251321

[6] Klimontovich Y L and Luzgin S N 1979 JETP Lett. 30610

[7] Saffman M 1998 Phys. Rev. Lett. 8165

[8] Wallis H 1997 Phys. Rev. A 562060

Krutitsky K V, Burgbacher F and Audretsch J 1999 Phys. Rev. A 591517

[9] Giovanazzi S, O’Dell D and Kurizki G 2002 Phys. Rev. Lett. 88130402

O'Dell D, Giovanazzi S, Kurizki G and Akulin V M 2000 Phys. Rev. Lett. 845687

[10] Avetisyan Yu A and Trifonov E D 2008 JETP 106426 Kurizki G, Mazets I E, O'dell D and Schleich W P 2004 Int. J. Mod. Phys. B 18961

[11] Cattani F, Anderson D, Kim A and Lisak M 2005 JETP Lett. 81561

[12] Helmholtz v 1881 Wied. Ann. 13385

[13] Landau L D and Lifshitz E M 1960 Electrodynamics of Continuous Media (New York: Pergamon)

[14] Pitaevskii L P 1961 Sov. Phys._JETP 391008

[15] Born M and Wolf E 1959 Principles of Optics (London: Pergamon)

[16] Balykin V I, Minogin V G and Letokhov V S 2000 Rep. Prog. Phys. 631429

[17] Dalfovo F, Giorgini S, Piatevskii L P and Stringari S 1999 Rev. Mod. Phys. 71 463-512

Leggett A J 2001 Rev. Mod. Phys. 73 307-56

[18] Cattani F, Geyko V, Kim A, Anderson D and Lisak M 2010 Phys. Rev. A (submitted)

[19] Marcuse D 1981 Appl. Opt. 203573

Iwashita K, Nakagawa K, Nakano Y and Suzuki Y 1982 Electron. Lett. 18873

[20] Agrawal G P 1995 Nonlinear Fiber Optics (London: Academic)

[21] Parent A, Morin M and Lavigne P 1992 Opt. Quantum Electron. 241071

Bagini V et al 1996 J. Opt. Soc. Am. A 131385

[22] Weiner J, Bagnato V S, Zilio S and Julienne P S 1999 Rev. Mod. Phys. 711

[23] Fioretti A, Amiot C, Dion C M, Dulieu O, Mazzoni M, Smirne G and Gabbanini C 2001 Eur. Phys. J. D 15 189-98 